\title{
Erratum to: Continuity, segmentation and faulting type of active fault zones of the 2016 Kumamoto earthquake inferred from analyses of a gravity gradient tensor
}

\author{
Nayuta Matsumoto $^{1 *}\left(\mathbb{0}\right.$, Yoshihiro Hiramatsu ${ }^{2}$ and Akihiro Sawada ${ }^{2}$
}

\section{Erratum to: Earth, Planets and Space (2016) 68:167 DOI 10.1186/s40623-016-0541-y}

After the publication (Matsumoto et al. 2016), we found a mistake in calculation of $\beta$ shown in Fig. 7 , which gave smaller values of $\beta$. However, this correction has not changed our discussion. Revised Fig. 7 has shown that the distribution of $\beta$ across the Futagawa and Hinagu

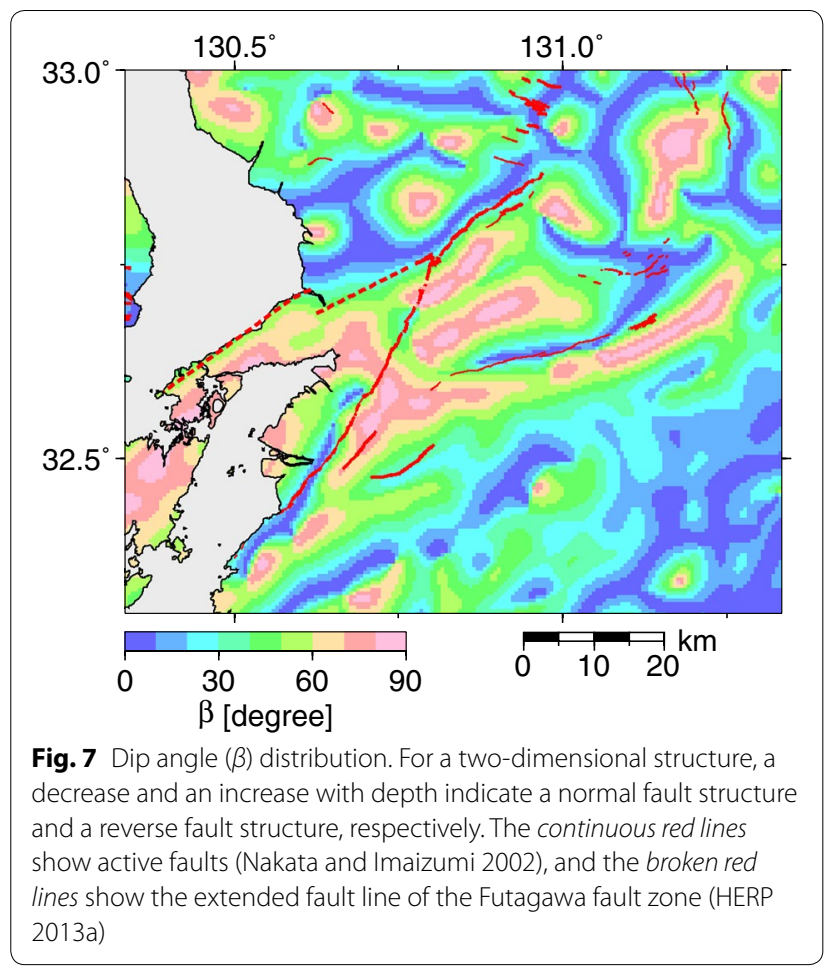

\footnotetext{
*Correspondence: n.matsumoto@stu.kanazwa-u.ac.jp

${ }^{1}$ Graduate School of Natural Science and Technology, Kanazawa

University, Kakuma, Kanazawa 920-1192, Japan
}

Full list of author information is available at the end of the article segments decreases from the south to the north, which implies normal faulting. It was also noticed that the author name "Hiramatsu Yoshihiro" is incorrect and should appear as "Yoshihiro Hiramatsu".

\section{Author details}

${ }^{1}$ Graduate School of Natural Science and Technology, Kanazawa University, Kakuma, Kanazawa 920-1192, Japan. ${ }^{2}$ School of Natural System, College of Science and Engineering, Kanazawa University, Kakuma, Kanazawa 920-1192, Japan.

The online version of the original article can be found under doi:10.1186/s40623-016-0541-y.

Received: 15 March 2017 Accepted: 15 March 2017

Published online: 27 March 2017

\section{Reference}

Matsumoto N, Hiramatsu Y, Sawada A (2016) Continuity, segmentation and faulting type of active fault zones of the 2016 Kumamoto earthquake inferred from analyses of a gravity gradient tensor. Earth Planets Space 68:167. doi:10.1186/s40623-016-0541-y 